\title{
La enseñanza de la conversación en ELE: Estado de la cuestión y perspectivas de futuro
}

\author{
Ana M. ${ }^{a}$ Cestero Mancera \\ Universidad de Alcalá \\ anam.cestero@uah.es
}

Resumenः El desarrollo teórico y metodológico que se ha producido en las últimas décadas en la Lingüística Aplicada a la adquisición y enseñanza de lenguas extranjeras ha destacado la parcela de la competencia comunicativa que tiene que ver con la interacción y, más concretamente, la que ha comenzado a llamarse «competencia conversacional». Los conocimientos que tenemos en la actualidad acerca del funcionamiento de la conversación española son bastante completos, por lo que es posible diseñar planes curriculares que atiendan a contenidos conversacionales; sin embargo, son muy pocos los trabajos realizados con respecto a diferencias culturales en la estructuración de la conversación y a las características que presenta la conversación en LE, así como a la enseñanza de mecanismos y fenómenos conversacionales. En los últimos años se ha podido avanzar en estos temas gracias a las investigaciones llevadas a cabo desde el Análisis de la Conversación, y, especialmente en el ámbito de ELE, a las realizadas por masterandos y doctorandos de programas especializados en enseñanza de ELE. En este trabajo se ofrece un estado de la cuestión de la investigación y enseñanza de la conversación en ELE y se apuntan las perspectivas de futuro que permitirán avances necesarios.

Palabras clave: Análisis de la conversación; Competencia conversacional; Mecanismos y fenómenos conversacionales; Adquisición y enseñanza de lenguas extranjeras.

Abstract: The theoretical and methodological development that has taken place in recent decades in Applied Linguistics on the acquisition and teaching of foreign languages has emphasized the place of the communicative competence that is related to interaction, currently known as «conversational competence». We already have extensive knowledge on how Spanish conversation works, so as to al- 
low us to design curricula that address conversational content; however, there are still very few studies carried out on cultural differences in the structure and characteristics of conversation in SFL, or on the teaching of conversational mechanisms and phenomena. In recent years progress has been made on these issues thanks to research conducted in the Conversation Analysis field, more specifically in the field of SFL by students in master and doctoral programs specializing in SFL teaching. This paper provides a state of the art on research and teaching of SFL conversation and suggests future prospects that will ensure progress.

Keywords: Conversational Analysis; Conversational competence; Conversational mechanisms and phenomena; Acquisition and teaching of foreign languages. 


\section{Introducción}

Es por todos bien conocido que el desarrollo teórico y metodológico que se ha producido en las últimas décadas en la Lingüística Aplicada a la adquisición y enseñanza de lenguas extranjeras ha destacado la parcela de la competencia comunicativa que tiene que ver con la interacción y, más concretamente, la que ha comenzado a llamarse «competencia conversacional». Las razones fundamentales por las que la conversación y su enseñanza han alcanzado tal prioridad son dos: la consideración de que el objetivo principal de la enseñanza de una lengua debe ser conseguir que el aprendiz pueda comunicar y comunicarse correctamente y de forma apropiada, y la conversación es la forma más natural, habitual y espontánea de comunicación interactiva humana, y, además, la constatación de que únicamente a través de la interacción comunicativa oral y, más concretamente, de la práctica conversacional es posible adquirir gran parte de las estructuras sintácticas complejas de una lengua, la mayoría de sus elementos discursivos o conversacionales y las funciones y actos comunicativos lingüístico-pragmáticos.

Teniendo en cuenta estos datos, parece conveniente pensar que los profesionales en la enseñanza de lenguas extranjeras deben favorecer y procurar, prioritariamente, la adquisición de la competencia conversacional, a partir del presupuesto teórico de base de que participar en una conversación requiere tener la capacidad cognitiva y la competencia lingüística y no verbal necesarias para producir y comprender enunciados, pero, además, y fundamentalmente, requiere poseer la capacidad discursiva e interactiva suficiente para cooperar y negociar con otras personas en su construcción (Cestero 2005; García García 2005 y 2009).

\section{La organización estructural de la conversación}

El término conversación se utiliza, habitualmente, en el ámbito de la enseñanza de lenguas extranjeras, para aludir a cualquier actividad comunicativa en la que distintos participantes alternan al hablar o en la que los aprendices sencillamente hablan. Esto conlleva que las clases de conversación se conviertan en espacios o tiempos «de habla», en los que se realizan actividades más o menos interactivas (debates, presentación de temas, simulaciones de compra-venta, etc.), que, en su mayoría, se alejan de lo que es una verdadera conversación cotidiana, esencial en el proceso de adquisición de la competencia comunicativa.

La conversación es una secuencia de intervenciones, realizadas por interlocutores diferentes que tienen la misma categoría real o funcional, con una organización general determinada no convencionalizada ni planificada y con una organización pormenorizada no predeterminada, producida mediante el funcio- 
namiento de un mecanismo de alternancia de turnos específico, no controlado o dirigido por ningún participante concreto, y cuyo objetivo social es, en principio, la comunicación por la comunicación (Cestero 2000a y 2005; Gallardo 1993 y 1996). Se trata, así, de una unidad de interacción social, construida a través de una serie ordenada de turnos, formulados, de manera alternativa, por los participantes, que resulta muy diferente de otros tipos de interacción, como las transacciones (entrevistas, encuentros entre médicos y pacientes o profesores y estudiantes, actividades de compra-venta, gestiones en un banco o en una administración pública, etc.) y las interacciones institucionalizadas (debates, mesas redondas, coloquios, juicios...).

Durante mucho tiempo, se ha considerado como una forma «libre» de comunicación humana (Levinson 1989). Es cierto que se trata de la manera más «espontánea y natural» que tenemos los seres humanos de comunicarnos, pero ello no significa que no esté sujeta a determinadas reglas, cuyo cometido es posibilitar y garantizar un funcionamiento y una forma de producción adecuados. La conversación es un texto, una interacción y un tipo específico de actividad interactiva y, como tal, está sujeta a las reglas textuales básicas (se ha de mantener la coherencia y la adecuación, y procurar cohesión interna), a los principios de interacción (principios de cooperación y de cortesía) y a las reglas, principios y mecanismos que rigen su propia estructuración. Con respecto a estos últimos, muy poco tratados en el proceso de enseñanza y adquisición de una lengua segunda o extranjera, es necesario subrayar que toda conversación tiene una organización interna característica que determina su producción, en la que están implicados mecanismos, elementos y fenómenos estructurales básicos (mecanismo de alternancia de turnos, finalización de turno, inicio de turno, diferentes tipos de turnos, funciones de la interrupción, estrategias de toma de turno interruptiva, turnos de apoyo, distintos tipos de intercambios, secuencias y sus tipos, estructura general de la conversación y cambio de tema, etc.), que la distingue de otras actividades interactivas y obliga a cumplir con los preceptos de estructuración normalizados de cada lengua y cultura.

Toda conversación está estructurada jerárquicamente, de manera que sus unidades principales se van uniendo y combinando hasta constituir la actividad comunicativa completa.

Las unidades primarias son dos: una unidad básica estructural, el turno, y una acción recurrente o reiterativa estructuradora, la alternancia de unidades básicas.

Podemos definir el turno como un «período de tiempo que comienza cuando una persona empieza a comunicar y concluye cuando dicha persona deja de hacerlo»; en ese período de tiempo, el hablante emite un mensaje con intención de ofrecerlo completo. Una vez concluido el mensaje proyectado para un turno, 
el hablante calla y otro participante puede tomar la palabra, comenzar un turno; puede producirse, entonces, una alternancia.

La sucesión de dos turnos efectuados por participantes diferentes se denomina intercambio y constituye la segunda unidad de la estructura conversacional. Son los intercambios, o las alternancias de turnos, los que van produciendo el desarrollo de la actividad interactiva que nos ocupa.

Los distintos intercambios suelen agruparse en secuencias: sucesión de tres o más turnos con coherencia y cohesión temática o funcional, es decir, unidades conversacionales, formadas por tres o más turnos -dos intercambios o más-, que cumplen una única función principal determinada o presentan una relación temática o pragmática específica que marca, de alguna manera, su estructura interna.

Por último, la combinación determinada de diferentes secuencias (secuencia de apertura, secuencia o secuencias temáticas y secuencia de cierre) conforma la estructura general de la conversación.

En líneas generales, podemos decir que la conversación está organizada en dos niveles estructurales: un nivel local o pormenorizado, que conforma su microestructura, en el que se atiende a todos los fenómenos relacionados con los turnos, los intercambios y las secuencias (señales y tácticas de final de turno, marcadores de inicio de turno, interrupción, secuenciación adecuada de diferentes tipos de turnos, los turnos de apoyo, secuencias de distinto tipo - marco y temáticas-, etc.), y un nivel global, que conforma su macroestructura, que tiene que ver con su organización general, incluyendo en ella la organización temática y los cambios de tema. Estos dos niveles de estructuración sirven para guiar la presentación de las características conversacionales que deben recogerse en los diseños curriculares de enseñanza de lenguas segundas o extranjeras, pues parten de la unidad mínima y prosiguen, de forma ordenada, hasta llegar a la unidad de comunicación completa. Es este el camino, además, que debe seguirse al enseñar las características conversacionales, ya que, a nuestro modo de ver, debe programarse una enseñanza gradual y progresiva, comenzando con los mecanismos, fenómenos y elementos de la microestructura y avanzando hasta llegar a los de la macroestructura, procediendo de la misma forma en cada uno de los niveles de enseñanza/aprendizaje.

Por razones de espacio, no podemos describir y explicar exhaustivamente aquí cómo se organiza y se produce la conversación española y cuáles son los mecanismos, elementos y fenómenos que la caracterizan. Los datos que acabamos de ofrecer no pretenden otra cosa que situar, de forma precisa, en un objeto concreto, la estructuración de la conversación, con el fin de crear un marco de 
referencia que permita entender la situación actual de la enseñanza de la conversación en español como lengua extranjera (ELE) y las perspectivas de futuro que presentamos a continuación y que se han establecido a partir de la revisión de las investigaciones realizadas recientemente en el ámbito de la lingüística aplicada a la enseñanza de ELE.

\section{La enseñanza de la conversación en ELE: situación actual}

Para poder darle a la conversación la importancia que se merece en el ámbito de la enseñanza de lenguas extranjeras, ha de conocerse y tenerse en cuenta previamente qué es, cómo se produce, cómo funciona y cómo se estructura, además de cuáles son las diferencias sustanciales que se dan entre diferentes lenguas y culturas y por qué se caracteriza una conversación en lengua extranjera (LE).

Los conocimientos que tenemos en la actualidad acerca del funcionamiento de la conversación española son bastante completos (Briz 1996 y 1998; Cestero 2000a, 2000b y 2005; Gallardo 1993, 1996 y 1998; Tusón 1997), por lo que es posible diseñar sin dilación planes curriculares que atiendan a contenidos conversacionales; sin embargo, son muy pocos los estudios realizados con respecto a diferencias culturales en la estructuración de la conversación y a las características que presenta la conversación en LE. En los últimos años se ha podido avanzar en estos temas gracias a las investigaciones llevadas a cabo desde la corriente conocida como Análisis del discurso, en general, y desde la disciplina denominada Análisis de la Conversación, en particular (Jefferson 1992; Levinson 1989; Schiffrin 1994; Cestero 2005; Ambjoen 2008), y, especialmente en el ámbito de ELE, a la labor de investigación realizada por masterandos y doctorandos de programas especializados en enseñanza de ELE (García Castro 2003 y 2004; García García 2004, 2005, 2007 y 2009; Rubio 2007 y 2008; de Mingo 2008; Pérez 2009 y 2011; Martínez 2009; López Sáez del Burgo 2009; Inglés 2010; Sanz 2010; Pascual 2011 y López de Lerma 2011) como reseñaremos más adelante.

El postulado básico del Análisis de la conversación y de todos los estudios sobre la forma de comunicación más natural y habitual es que la conversación no debe concebirse como una unidad lingüística superior a la oración, sino como una actividad comunicativa, un tipo de interacción social, que posee una estructura y unas unidades propias e independientes. Dentro de esta corriente se sitúan algunos trabajos, de los pocos existentes, dedicados a aplicar los conocimientos que hoy día tenemos sobre la conversación y su funcionamiento a la adquisición y la enseñanza de lenguas segundas y extranjeras (Scarcella 1983 y 1988; Stewner- 
Manzanares 1983; Riggenbach 1991 y 2000; Gadner 1994 y 1997; Gardner y Wagner 2004, Briz 2004; Cestero 2005; Fernández y Albelda 2008, García Castro 2003, García García 2009, Rubio 2008, Pérez 2009 y 2011, Martínez 2009, Inglés 2010, Pascual 2011, entre otros). En relación a ello, cabe decir que los hallazgos de los analistas de la conversación se han tenido en cuenta para investigar cuatro temas relacionados con la adquisición de lenguas extranjeras (Scarcella 1988): la importancia de la conversación en el desarrollo sintáctico de la segunda lengua o lengua extranjera, las modificaciones conversacionales que realizan los nativos para facilitar la adquisición de lenguas extranjeras o segundas, la utilización de algunas estrategias de comunicación y el desarrollo de la adquisición de la competencia conversacional. Por otro lado, se ha trabajado, desde un enfoque interdisciplinar, en el estudio de características y estrategias conversacionales que difieren en distintas culturas y, por tanto, requieren una enseñanza programada en el aula de LE. Y, por último, se han realizado algunos trabajos de corte teórico o práctico que se centran en la didáctica de la conversación a partir de cuatro objetivos específicos (cfr. Scarcella 1988: 78 y ss.): reflexionar sobre la posibilidad de enseñar las características conversacionales explícitamente o de forma indirecta, fomentar y favorecer el uso de materiales lingüísticos auténticos, crear actividades de clase que favorezcan la aparición de contextos de aprendizaje óptimos y programar secuencias de actividades para trabajar la estructuración de la conversación. Presentamos, a continuación, el estado de la cuestión y las perspectivas de futuro de estos temas de estudio en el ámbito de ELE.

\subsection{Conversación y adquisición de lenguas extranjeras}

Como acabamos de mencionar, en lo que respecta a la adquisición de la conversación en el marco de aprendizaje de lenguas segundas y extranjeras, se ha trabajado, de forma general y en ámbito internacional, en torno a cuatro temas fundamentales: la importancia de la conversación en el desarrollo sintáctico de la segunda lengua o lengua extranjera, las modificaciones conversacionales que realizan los nativos para facilitar la adquisición de lenguas extranjeras o segundas, la utilización de algunas estrategias de comunicación y el desarrollo de la adquisición de la competencia conversacional.

Con relación al primero de los temas, parece estar constatado, como se apuntaba al principio, que el desarrollo de la segunda lengua o la lengua extranjera, especialmente del componente sintáctico, se produce a través de la práctica conversacional, $y$, por lo tanto, se facilita ofreciendo a los estudiantes oportunidades de producir las formas o funciones aprendidas en conversación espontánea y lo más natural posible. El procedimiento, según Hatch (1978ः 404), es el siguiente: 
el alumno va aprendiendo cómo realizar la conversación, cómo interactuar verbalmente, a medida que va necesitando que su lenguaje sea más comprensible o que su mensaje sea entendido, a medida que va necesitando comunicarse más productivamente, $y$, a partir de la práctica conversacional o interactiva, se van desarrollando determinadas estructuras sintácticas, especialmente las más complejas (Ellis 1999).

Por otro lado, se ha demostrado que las modificaciones lingüísticas y conversacionales que hacen nativos y profesores cuando interactúan con un extranjero no son ocasionales ni involuntarias, sino que son necesarias y extraordinariamente útiles para facilitar la adquisición de lenguas segundas o extranjeras. Esta forma de uso de la lengua materna se considera un registro específico, relacionado con el foreigner talk, que, aunque se caracteriza por la simplificación, tiene como rasgo definitorio un conjunto de señales conversacionales (preguntas de confirmación y de clarificación, elementos fáticos de control de contacto y de comprensión, repeticiones totales o parciales, etc.) que ayudan a la adquisición de la lengua, a la vez que involucran al aprendiz, en mayor medida, en el proceso de aprendizaje.

Las estrategias comunicativas han sido uno de los temas más investigados en los últimos años y con más aplicaciones a la enseñanza de segundas lenguas y lenguas extranjeras, si bien es cierto que no todos los tipos de estrategias han tenido el mismo tratamiento y que aún son muchos los que no se conocen con cierta profundidad. De los estudios realizados se desprende que los aprendices de lenguas extranjeras que adquieren estrategias comunicativas pronto y de forma progresiva se manejan mejor y con más fluidez y soltura, en conversación, que los que no lo hacen, con las consecuencias que ello tiene en la adquisición de la competencia lingüística y comunicativa; lo que hace no ya recomendable, sino necesaria, la atención específica a estos elementos en los programas de enseñanza de lenguas segundas y extranjeras.

Por último, se ha intentado conocer el desarrollo de «indicios específicos conversacionales» en los aprendices de lenguas (Scarcella 1983). A este respecto, queda aún mucho por hacer, pero las investigaciones realizadas indican que parece haber patrones en la adquisición de algunas características conversacionales; así, por ejemplo, ciertas secuencias o partes de secuencias, como los saludos, se adquieren pronto y sin mucha dificultad, mientras que otras, como las secuencias de cierre o los precierres, tardan bastante en adquirirse y conllevan mucha dificultad.

Son muy pocos los trabajos realizados sobre estos temas en relación a la enseñanza de ELE, pero los que tenemos son fundamentales para seguir avanzando. Cabe destacar la investigación de M. García (2009), en la que se demuestra que 
los marcadores de señalización y relación conversacionales (señales de inicio y final de turno, marcas de cambio o retroceso temático, etc.) son elementos lingüísticos que tienen una gran dificultad de aprendizaje, para los estudiantes alemanes de ELE.

Por su parte, los estudios realizados por M. Rubio (2008) revelan que, en la conversación en ELE de estudiantes taiwaneses, se observa un número excesivo de interrupciones involuntarias que parecen indicar un uso distinto del mecanismo de alternancia de turnos o una interpretación errónea de lugares apropiados para el cambio de hablante y, además, las verbalizaciones usadas por los taiwaneses en las conversaciones en ELE son transferidas del chino.

Por último, J. Pérez $(2009,2011)$ ha investigado en profundidad la producción de turnos de apoyo en la conversación en ELE de estudiantes taiwaneses, a partir de las evidencias de diferencias transculturales halladas previamente sobre este fenómeno en el marco del análisis de la conversación ${ }^{1}$, y ha constatado que los estudiantes taiwaneses utilizan estrategias comunicativas propias de su lengua materna. Las transferencias documentadas por Pérez tienen que ver, especialmente, con la producción frecuente de los apoyos en lugares apropiados para la transición y con el uso de apoyos enfáticos. Los estudiantes británicos de ELE, sin embargo, según documenta Inglés (2010) en un estudio similar al de Pérez (2009), suelen transferir la proporción de uso de turnos de apoyo cuando conversan en español, no muestran una variedad aceptable de apoyos diferentes y recurren frecuentemente a la lengua materna para producir apoyos. Y, por último, los estudiantes italianos de ELE presentan en sus conversaciones en lengua meta una proporción anómalamente alta de turnos de apoyo y de secuencias especiales con apoyos, lo que podría considerarse transferencia susceptible de fosilización (Pascual 2011).

\subsection{Diferencias culturales en la interacción conversacional}

Relacionado con el desarrollo de las características conversacionales y su pronta o tardía adquisición, además de la menor o mayor dificultad, está su universalidad. Los estudios pioneros realizados sobre el tema indicaban que, aunque existen tendencias universales para muchos aspectos de la conversación, que, indudablemente, ayudan al aprendiz a conversar y adquirir una lengua no materna, existen otras que no lo son y difieren en forma o función (marcas de final de turno, interrupción, apoyos conversacionales, secuencia de cierre conversacional, etc.; véase Cestero 2005), y, dado que los alumnos no comienzan la empresa de adquirir una lengua segunda o extranjera equipados con el conocimiento de las normas y reglas conversacionales de la lengua objeto de estudio, sino con el de su lengua

$\overline{1 . S . ~ K . ~ M a y n a r d ~(1990) ~ y ~ H . ~ T a o ~ y ~ S . ~ A . ~ T h o m p s o n ~(1991) . ~}$ 
materna, generalmente experimentan dificultades al interactuar con nativos, que, en muchas ocasiones, dificultan o impiden la intercomunicación y, consecuentemente, la adquisición de la competencia comunicativa completa. Esta es la razón principal por la que la conversación y sus unidades estructurales básicas deben ser atendidas, de forma adecuada, en el proceso de enseñanza y aprendizaje de lenguas extranjeras.

Hasta ahora son escasas las investigaciones centradas en este tema, pero poco a poco vamos adelantando camino y, además, de forma segura, pues los conocimientos obtenidos provienen de investigaciones empíricas de gran envergadura. En los años setenta del siglo $x x$, comenzaron a realizarse estudios sobre la conversación en lenguas y culturas diferentes de la anglo-americana ${ }^{2}$ y rápidamente aparecieron datos que demostraban la influencia cultural sobre la organización de la interacción verbal y no verbal ${ }^{3}$.

Partiendo de estos estudios ya clásicos sobre diferentes lenguas y culturas, se han realizado y se están llevando a cabo investigaciones enmarcadas en el ámbito de la lingüística aplicada a la enseñanza del español como lengua extranjera. En ellas se investiga cómo producen la conversación en español estudiantes de español como lengua extranjera de diferentes nacionalidades y culturas, la influencia que las características conversacionales de la lengua materna tiene sobre la producción de conversación, qué fenómenos o elementos conversacionales resultan anómalos, etc. y, con ello, se trata de conocer cuáles son las características conversacionales que necesitan una enseñanza programada en el aula de ELE y cómo debe abordarse dicha enseñanza.

El trabajo más completo de los realizados hasta ahora es el de M. García (García García 2009), que tiene en consideración hallazgos de un trabajo previo, de 2004, para estudiar la alternancia de turnos y el manejo de la agenda en

2. I. Daden (1982), J. Haviland (1977), R. Hopper (1990-91), M. Moerman (1972, 1977 y 1988). Es importante el estudio de M. Moerman, que constata similitudes y diferencias en la conversación de tailandeses y americanos. El autor apunta que los motivos de ciertas variaciones están en valores culturales y señala que tales diferencias más que «sintácticas» son «semánticas». Estamos de acuerdo con él en que debería desarrollarse más este tipo de estudios utilizando para ello la metodología, técnicas y hallazgos del Análisis de la Conversación. Hay otros investigadores que trabajan en este campo, pero sus estudios están aún sin publicar, entre ellos se encuentran, P. Brown y S. Levinson.

3. Véase a este respecto el estudio que E. M. Albert (1972) hace sobre el sistema de alternancia de turnos en el burundi, en el que descubre que en el mencionado pueblo africano la alternancia de turnos está preasignada atendiendo al rango de los participantes, dando lugar a un orden de turnos preestablecido, como ocurre en las culturas anglohablantes en contextos institucionales. Es interesante, también, el trabajo de D. Tannen (1983 y 1987) acerca del habla simultánea, en el que, analizando el estilo conversacional de nativos de diferentes ciudades, relacionados con culturas distintas, llega a la conclusión de que la superposición, más allá de suponer una violación del sistema de toma de turno, es una de las características más sobresalientes, valoradas e incluso requeridas, del «estilo de gran envolvimiento» que presentan algunos hablantes. Como ejemplo de no universalidad del mecanismo de alternancia de turnos de habla propuesto por Sacks, Schegloff y Jefferson (1974), véase también J. Bilmes (1992), R. Hayashi (1991) y L. Philips (1976). 
las conversaciones en ELE de estudiantes alemanes. Las conclusiones a las que llegó la autora en su investigación muestran una caracterización conversacional en ELE que difiere de la de la lengua meta, en la que se destaca, con respecto a la alternancia de turnos, la frecuencia de turnos más cortos de lo habitual en español, cuando hay una alta implicación de los interlocutores, y de turnos excesivamente largos, cuando se establece una relación de desigualdad entre ellos, lo que indica un predominio de atención a la forma y producción en lugar de a la cooperación; la frecuencia de alternancias propias es muy alta (en torno al 75\%), en comparación con lo que es normal en español (50\%), e indica un predominio de respeto ajeno, y, por último, el uso de marcadores de inicio es escaso y se prefiere la risa como marca de cesión de turno. En lo que respecta al control temático, es significativo el poco uso de recursos para introducir temas, lo que provoca falta de cohesión general, el inicio preferente de temas mediante preguntas y el cierre de temas con silencio, así como las dificultades para reintroducir y retomar temas y para controlar el equilibrio en el desarrollo de temas en conversación. Estas características conversacionales distan del comportamiento cooperativo y negociador propio de la conversación en español.

Por su parte, M. Rubio ha iniciado el estudio de la interrupción en la conversación en ELE de estudiantes taiwaneses. En un estudio exploratorio (Rubio 2007), concluyó que la caracterización de la conversación en ELE resulta anómala por el bajo número de interrupciones que se producen, así como por la escasa aparición de superposición de habla — normalmente colaborativa- y por el uso o la interpretación frecuentes de vacíos y pausas como señales de final de turno, lo que apunta la existencia de un patrón de comportamiento poco disruptivo y, en relación a la función de la interrupción en español, poco colaborador, en el que el interlocutor está preocupado, fundamentalmente, por construir su turno, si bien no se produce competitividad.

El trabajo de M. C. Martínez (2009) es también preliminar, pero nos informa de cómo manejan la organización temática estudiantes finlandeses de ELE. Según los análisis realizados, la linealidad y coherencia temáticas, así como la articulación de secuencias temáticas por parte de las estudiantes de ELE no resultan incorrectas, aunque debe estudiarse cómo se producen en la conversación en lengua española para comprobar si resultan adecuadas; precisamente esta es la investigación que está llevando a cabo en estos momentos, así que debemos esperar a que la termine para conocer más datos.

Por último, la vía de investigación más fructífera hasta ahora es la que inició J. Pérez en 2009 con el estudio de los turnos de apoyo en la conversación de estudiantes taiwaneses de ELE. En su tesis doctoral, Pérez (2011) demuestra que 
es característica de la conversación en ELE de taiwaneses la producción de apoyos verbales en lugares apropiados para el cambio de hablante, lo que indica un patrón de comportamiento respetuoso y poco intrusivo, la existencia de un componente enfático en la emisión de los apoyos transferido de la lengua y cultura maternas, y la utilización frecuente de apoyos de reafirmación y entendimiento, de forma que los interlocutores se aseguran el entendimiento del enunciado en marcha. Tomando como marco teórico y metodológico los trabajos de Pérez, B. Inglés (2010) y C. Pascual (2011) han analizado los turnos de apoyo conversacionales producidos por estudiantes británicos e italianos de ELE, respectivamente. Aunque se trata de estudios exploratorios, nos han permitido saber que los estudiantes británicos de ELE, cuando conversan en español, contrariamente a como ocurre en la conversación española, no presentan una frecuencia alta de producción de apoyos y, en su mayoría, son requeridos pragmáticamente o interaccionalmente y no voluntarios, hacen más uso de apoyos de entendimiento que de apoyos de acuerdo y de apoyos compuestos que de apoyos complejos, lo que caracteriza su interlengua, y cuentan con un uso afectivo de los turnos de apoyo que favorece el acercamiento social y la empatía. Por su parte, los estudiantes italianos de ELE producen más turnos de apoyo y secuencias con apoyo, en conversación en ELE, que los españoles, los taiwaneses y los ingleses y, como los dos últimos, emiten un gran número de apoyos de entendimiento característicos de la interlengua.

Los trabajos que acabamos de mencionar permiten afianzar nuestro conocimiento sobre diferencias transculturales en la producción de conversación y, lo que es más importante en el tema que nos ocupa, nos han permitido conocer la existencia de diferencias básicas en la producción conversacional en ELE de estudiantes procedentes de distintas culturas, que dificultan la adquisición de la lengua extranjera o producen una adquisición anómala. La identificación de tales diferencias permite diseñar de forma más adecuada el plan docente para la enseñanza de la conversación en español y, por tanto, predice un avance considerable y de gran importancia en la enseñanza de ELE. En los próximos años se han de seguir las líneas de estudio comenzadas, ampliando el ámbito de investigación con estudiantes procedentes de culturas distintas a las ya tratadas (se ha emprendido el estudio de los turnos de apoyo en estudiantes de ELE árabes y japoneses), y se han de abrir nuevas líneas de investigación sobre fenómenos conversacionales aún no tratados (marcas de final de turno, secuencias de cierre conversacional, etc.). Además, se ha de dedicar un gran esfuerzo al ámbito de la didáctica, ya que, como revisaremos a continuación, aún está por desarrollar. 


\subsection{La enseñanza de la conversación}

En las organizaciones docentes y en los diseños curriculares actuales, la conversación ocupa un lugar destacado, e, incluso, en muchos de ellos, se destina un número similar de clases u horas a trabajar la competencia lingüística y la conversacional, haciéndolo por separado, aunque sin perder de vista la interrelación existente entre ambas competencias. A pesar de ello, las clases de conversación no son todo lo productivas que debieran y podrían ser, pues suelen considerarse complementarias de las de gramática y, por lo tanto, menos importantes; esa supuesta complementariedad hace que su contenido esté, a lo sumo, relacionado con el que se trabaja en gramática o léxico o, en los casos en que se opte por un enfoque comunicativo o nociofuncional, con estrategias, elementos y fenómenos relacionados con las reglas de producción textual o de interacción. Además, las actividades realizadas en estas clases van encaminadas a hacer hablar al alumno, a expresar ideas, gustos, preferencias, juicios... enunciados y no a hacerle interactuar de forma natural; así, se trabaja, sobre todo, con interacciones transaccionales o con simulaciones de conversaciones cotidianas no sujetas a los principios naturales de estructuración, con debates, con exposiciones de temas, etc., con los que, si bien se favorece la adquisición de reglas y normas de distintos tipos de textos, no se promueve la adquisición de la «competencia conversacional».

No obstante, para que los especialistas en enseñanza de lenguas extranjeras puedan trabajar de forma adecuada en la didáctica de la conversación, es necesario contar con conocimientos básicos sobre la estructuración conversacional y los mecanismos, elementos y fenómenos relacionados con ella y, como hemos mencionado con anterioridad, tales conocimientos no se han tenido hasta muy recientemente y se está investigando en estos momentos las diferencias en la producción de la conversación de aprendices de ELE de diferentes culturas. Es, por tanto, al retraso en el desarrollo del estudio del «habla», en general, y del análisis de la conversación, en particular, al que debemos achacar la escasa aparición de los aspectos estructurales de la conversación en los diseños curriculares y en los materiales de enseñanza de lenguas extranjeras y, consecuentemente, el tratamiento erróneo que tiene esta actividad interactiva en el aula de lenguas extranjeras.

\subsubsection{La conversación en los programas de enseñanza de lenguas extranjeras}

La planificación docente y curricular se lleva a cabo de forma específica e independiente en los diferentes centros especializados en enseñanza de lenguas extranjeras, lo que impide que podamos generalizar sobre el tratamiento que la conversación y sus unidades estructurales recibe, pero, desde hace unos años, 
contamos, para el ámbito europeo, con un documento que aporta una base teórico-metodológica única para el aprendizaje, la enseñanza y la evaluación de las lenguas: el Marco común europeo de referencia para las lenguas: aprendizaje, enseñanza, evaluación, elaborado por el Consejo de Europa (Consejo de Europa 2002). En el documento al que hacemos mención, que adopta un enfoque orientado a la acción, se da prioridad a la competencia comunicativa, lo que favorece y promueve la inclusión de mecanismos, elementos y fenómenos estructurales conversacionales entre los contenidos establecidos como base.

Con respecto a los aspectos relacionados con la producción de la conversación, el Marco común europeo de referencia para las lenguas los atiende al tratar la competencia sociolingüística y, especialmente, la competencia pragmática, en la que distingue tres subtipos relacionados de alguna manera con la estructuración de la conversación:

a) Competencia discursiva: es la que permite organizar, estructurar y ordenar los mensajes.

b) Competencia funcionalः gracias a la cual cumplimos funciones comunicativas con los mensajes que utilizamos.

c) Competencia organizativa: mediante ella podemos secuenciar los mensajes según esquemas de interacción y de transacción.

De la competencia discursiva atañe a los contenidos que nos competen los siguientes: la capacidad de estructurar y controlar el discurso en función de la organización temática y la organización del texto (estructuración de la información en la realización de las distintas secuencias).

De la competencia funcional nos concierne, de manera específica, la estructuración del discurso — función 1.5, con sus 28 microfunciones: comienzo, turnos de habla, conclusión... - (Consejo de Europa 2002:123).

De la competencia organizativa nos afectan los esquemas-modelos de interacción social (concretamente el de la conversación casual), aunque no aparecen desarrollados completamente en el trabajo que comentamos.

El Marco común europeo de referencia para las lenguas recoge, así, bastantes de los aspectos conversacionales que tienen que ver con la producción de la conversación, si bien aparecen dispersos por toda la obra, distribuidos en distintos componentes y sin el detalle y la profundidad que una planificación específica requiere.

El Instituto Cervantes sacó a la luz, en 2006, un nuevo Plan Curricular, en el que recoge y desarrolla los niveles comunes de referencia establecidos por el Marco común europeo (Instituto Cervantes 2006). Presenta, como novedades de la actualización, el «desarrollo de contenidos en una dimensión supraoracional, de carácter pragmático-discursivo: funciones lingüísticas, aspectos pragmáticos 
y géneros discursivos» (Instituto Cervantes 2006: 30). Así, los contenidos relacionados con la organización de la conversación que tratamos aquí aparecen recogidos en los apartados correspondientes al componente pragmático-dicursivo: apartado 5 (funciones), apartado 6 (tácticas y estrategias pragmáticas) y apartado 7 (géneros discursivos y productos textuales), de los seis niveles de referencia ${ }^{4}$ (3); sin embargo, seguimos sin encontrar el desarrollo y el detalle que los mecanismos, fenómenos y elementos estructurales requieren y, una vez más, están dispersos por distintos contenidos, lo que, a nuestro modo de ver, no favorece la enseñanza de las características básicas de la conversación.

No podemos ofrecer aquí un inventario y una descripción detallados de todos los aspectos estructurales que están implicados en la producción de la conversación, tarea compleja e interdisciplinaria que aún está por hacerse en el marco de la Lingüística, pero, a partir de nuestra experiencia y de las ideas presentadas previamente, y teniendo en cuenta las recomendaciones del Marco común europeo de referencia para las lenguas (Consejo de Europa 2002), sí podemos establecer los mecanismos, fenómenos y elementos propios de la estructuración conversacional que deben ser tenidos en cuenta en los diferentes diseños curriculares por requerir una enseñanza específica, completando ampliamente los contenidos que aparecen en el Plan Curricular del Instituto Cervantes (Instituto Cervantes 2006). Organizados por niveles y recogidos en forma de objetivos-contenidos, son los siguientes:

4. Los aspectos estructurales de la conversación que recoge el Plan curricular del Instituto Cervantes (Instituto Cervantes 2006), expuestos de forma sintética, son los siguientes:

Funciones (5)

Estructurar el discurso: establecer la comunicación y reaccionar, saludar y responder a un saludo, preguntar por una persona y responder, pedir una extensión o habitación y responder, preguntar si se puede dejar un recado, preguntar por el estado general de las cosas y responder, solicitar que comience un relato y reaccionar, introducir un tema del relato y reaccionar, indicar que se sigue el relato con interés, controlar la atención del interlocutor, introducir un hecho, organizar la información, conectar elementos, reformular lo dicho, destacar un elemento, introducir palabras de otros, citar, abrir una digresión, cerrar una digresión, rechazar un tema o aspecto del tema, interrumpir, indicar que se puede reanudar el discurso, pedir a alguien que guarde silencio, conceder la palabra, indicar que se desea continuar el discurso, concluir el relato, introducir un nuevo tema, proponer el cierre, aceptar el cierre, rechazar el cierre introduciendo un nuevo tema y despedirse y responder a despedidas.

Tácticas y estrategias pragmáticas (6)

Construcción e interpretación del discurso: mantenimiento del referente y del hilo discursivo, marcadores del discurso

Géneros discursivos y productos textuales (7)

Muestras de géneros: conversación transaccional. Macrofunciones: macrofunción descriptiva, macrofunción narrativa, macrofunción expositiva, macrofunción argumentativa.

Además, como ocurría en el Plan curricular de 1994 y como pasa en el Marco común europeo de referencia para las lenguas, aparecen otros aspectos estructurales de la conversación dispersos por apartados correspondientes a otros contenidos funcionales, culturales, etc. 


\section{A. Usuario básico}

\section{A.1. Acceso}

- Producción y reconocimiento de turnos de habla breves completos.

- Producción y reconocimiento de algunas tácticas de conclusión de turno.

- Producción y reconocimiento de algunas indicaciones de toma de turno.

- Entendimiento, producción y reconocimiento de apoyos conversacionales de uso muy frecuente requeridos pragmáticamente.

- Entendimiento, producción y reconocimiento de intercambios breves cooperativos pragmáticamente.

- Entendimiento, producción y reconocimiento de secuencias de apertura sencillas.

- Entendimiento, producción y reconocimiento de secuencias de cierre sencillas.

* Producción de conversaciones cotidianas completas muy breves (variando contextos).

\section{A. 2. Plataforma}

- Producción y reconocimiento de turnos de habla breves completos.

- Producción y reconocimiento de más tácticas de conclusión de turno.

- Producción y reconocimiento de más indicaciones de toma de turno.

- Entendimiento, producción y reconocimiento de apoyos conversacionales de uso muy frecuente requeridos pragmática o interaccionalmente.

- Entendimiento, producción y reconocimiento de intercambios breves cooperativos pragmáticamente.

- Entendimiento, producción y reconocimiento de secuencias de apertura sencillas.

- Entendimiento, producción y reconocimiento de secuencias de cierre sencillas.

* Producción de conversaciones cotidianas completas muy breves (variando contextos).

\section{B. Usuario independiente}

\section{B.1. Umbral}

- Producción y reconocimiento de turnos de habla breves completos.

- Producción y reconocimiento de más tácticas de conclusión de turno.

- Producción y reconocimiento de más indicaciones de toma de turno.

- Entendimiento, producción y reconocimiento de apoyos conversacionales de uso muy frecuente requeridos pragmática o interaccionalmente. 
- Entendimiento, producción y reconocimiento de apoyos conversacionales de acuerdo y entendimiento de uso frecuente.

- Entendimiento, producción y reconocimiento de intercambios breves cooperativos pragmáticamente.

- Entendimiento, producción y reconocimiento de intercambios breves relacionados.

- Entendimiento, producción y reconocimiento de secuencias de apertura sencillas.

- Entendimiento, producción y reconocimiento de secuencias de cierre sencillas.

* Producción de conversaciones cotidianas breves completas (variando contextos).

\section{B.2. Avanzado}

- Producción y reconocimiento de turnos de habla completos.

- Producción y reconocimiento de más tácticas de conclusión de turno.

- Producción y reconocimiento de más indicaciones de toma de turno.

- Producción y reconocimiento de indicaciones de movimientos en el turno.

- Entendimiento y reconocimiento de algunas interrupciones justificadas.

- Entendimiento, producción y reconocimiento de apoyos conversacionales requeridos pragmática o interaccionalmente.

- Entendimiento, producción y reconocimiento de apoyos conversacionales de acuerdo y entendimiento.

- Entendimiento, producción y reconocimiento de intercambios breves cooperativos pragmáticamente.

- Entendimiento, producción y reconocimiento de intercambios breves relacionados.

- Reconocimiento de segundas partes no prioritarias en pares adyacentes.

- Entendimiento, producción y reconocimiento de secuencias de apertura completas.

- Entendimiento, producción y reconocimiento de secuencias de cierre completas.

- Entendimiento, producción y reconocimiento de secuencias de narración sencillas.

- Entendimiento, producción y reconocimiento de secuencias de inserción.

- Entendimiento, producción y reconocimiento de secuencias laterales.

- Entendimiento, producción y reconocimiento de estrategias sencillas para cambiar de tema.

* Producción de conversaciones cotidianas completas (variando contextos). 


\section{Usuario competente}

\section{C.1. Dominio operativo eficaz}

- Producción y reconocimiento de turnos de habla completos.

- Producción y reconocimiento de tácticas y de algunas marcas inferenciales de conclusión de turno.

- Producción y reconocimiento de indicaciones de toma de turno.

- Producción y reconocimiento de indicaciones de movimientos en el turno.

- Entendimiento y reconocimiento de interrupciones justificadas.

- Producción y reconocimiento de estrategias frecuentes de toma de turno interruptiva.

- Entendimiento, producción y reconocimiento de apoyos conversacionales requeridos pragmática o interaccionalmente.

- Entendimiento, producción y reconocimiento de apoyos conversacionales de acuerdo, entendimiento, seguimiento, conclusión, recapitulación y reafirmación de uso frecuente.

- Entendimiento, producción y reconocimiento de intercambios cooperativos pragmáticamente.

- Entendimiento, producción y reconocimiento de intercambios relacionados.

- Entendimiento, producción y reconocimiento de intercambios independientes sencillos.

- Producción y reconocimiento de segundas partes no prioritarias en pares adyacentes.

- Entendimiento, producción y reconocimiento de secuencias de apertura completas.

- Entendimiento, producción y reconocimiento de secuencias de cierre completas.

- Entendimiento, producción y reconocimiento de secuencias de narración.

- Entendimiento, producción y reconocimiento de secuencias de inserción.

- Entendimiento, producción y reconocimiento de secuencias laterales.

- Entendimiento, producción y reconocimiento de estrategias para cambiar de tema.

* Producción de conversaciones cotidianas completas (variando contextos).

\section{C.2. Maestría}

- Producción y reconocimiento de turnos de habla completos.

- Producción y reconocimiento de las marcas inferenciales de conclusión de turno.

- Producción y reconocimiento de indicaciones de toma de turno. 
- Producción y reconocimiento de indicaciones de movimientos en el turno.

- Entendimiento, producción y reconocimiento de interrupciones justificadas.

- Producción y reconocimiento de estrategias de toma de turno interruptiva.

- Entendimiento, producción y reconocimiento de apoyos conversacionales requeridos pragmática o interaccionalmente.

- Entendimiento, producción y reconocimiento de apoyos conversacionales de acuerdo, entendimiento, seguimiento, conclusión, recapitulación, reafirmación y combinados, guardando intervalos.

- Producción de distintos tipos de alternancias turno de habla-apoyo.

- Entendimiento, producción y reconocimiento de intercambios cooperativos pragmáticamente.

- Entendimiento, producción y reconocimiento de intercambios con turnos conectados sintáctico-semánticamente.

- Entendimiento, producción y reconocimiento de intercambios relacionados.

- Entendimiento, producción y reconocimiento de intercambios independientes.

- Producción y reconocimiento de segundas partes no prioritarias en pares adyacentes.

- Entendimiento, producción y reconocimiento de secuencias de apertura completas.

- Entendimiento, producción y reconocimiento de secuencias de cierre completas.

- Entendimiento, producción y reconocimiento de secuencias de narración.

- Entendimiento, producción y reconocimiento de secuencias de inserción.

- Entendimiento, producción y reconocimiento de secuencias laterales.

- Entendimiento, producción y reconocimiento de otros tipos de secuencias.

- Entendimiento, producción y reconocimiento de cambios de tema.

* Producción de conversaciones cotidianas completas (variando contextos).

Las clases de conversación, en los procesos de enseñanza de español como lengua extranjera, se programan con el objetivo general de hacer hablar al aprendiz, introduciéndole en contextos que pueden distar mucho de lo que se entiende por conversación (debates, exposiciones, transacciones varias, etc.), de ahí que en sus diseños curriculares, como contenidos específicos, solo aparezcan algunos de los fenómenos estructurales que aquí tratamos, que son abordados, además, de manera muy superficial y sin una base teórica firme. Este hecho se refleja claramente en la escasa aparición de los aspectos estructurales conversacionales en los manuales y materiales para la enseñanza de español como lengua extranjera edi- 
tados. Por norma general, en todos ellos se utiliza la conversación como actividad o espacio para trabajar la competencia lingüística o las funciones comunicativas, sin que ello implique la enseñanza específica de las reglas organizativas de la conversación y de los fenómenos lingüísticos y no verbales relacionados con ellas, ni el tratamiento efectivo de todas las funciones comunicativas implicadas en su estructuración (véase García Castro 2003 y López de Lerma 2011). No obstante, la excepción confirma la regla y, así, podemos apuntar unos pocos manuales en los que se trabajan aspectos conversacionales estructurales como son los siguientes: Curso intensivo de español. Ejercicios prácticos (Fente y otros 1982 y 1990), Planet@ (Cerrolaza y otros 1998 y 1999),Ven (Castro y otros 1990 y 1992), Sueña 3 (Álvarez y otros 2001) y Sueña 4 (Blanco y otros 2001) y, de forma especial, Hablamos español -niveles B y C- (Ridruejo, Hoyos, Mendizábal y Vela 2010; Álvarez, López y Simarro 2010). Con respecto a los materiales complementarios para la enseñanza de español como lengua extranjera, cabe destacar ELE. Tácticas de conversación (Varela 1991), Actos de babla de la lengua española (Fernández 1991), Repertorio de funciones comunicativas del español (Gelabert y otros 1996) y La destreza oral (Vázquez 2000). Estos últimos son los trabajos editados que más aspectos estructurales conversacionales recogen, si bien no contienen la totalidad de fenómenos y elementos que requieren una enseñanza específica y no cuentan con la que, a nuestro entender, es la distribución adecuada de elementos, ni con las explicaciones y prácticas que permitan a profesores y estudiantes trabajarlos en profundidad.

\subsubsection{Didáctica de la conversación en la clase de ELE}

La Conversación cotidiana es mucho más que una serie de intercambios de enunciados; en algunas lenguas, como la española, es una construcción conjunta de mensajes. Consiste en una serie de intervenciones comunicativas y estructurales que son producidas e interpretadas de acuerdo con una serie de mecanismos y reglas de interacción y estructuración adquiridas por el nativo en la etapa de socialización y puestas en práctica y entendidas de forma intuitiva y automática. El estudiante extranjero posee las de su propia lengua y cultura y, si no se le enseñan las de la lengua y cultura meta, las sustituirá por las suyas, fosilizándolas y no cambiándolas a pesar de su frecuente interacción con nativos. Como consecuencia, nunca conversará fluidamente en la lengua aprendida; si intenta hacerlo, obligará a su interlocutor a variar su estrategia o se sentirá frustrado por su escasa intervención. Para conseguir que los estudiantes adquieran «competencia conversacional» se ha de enseñar, por un lado, las reglas de interacción y los fenómenos 
y elementos relacionados con ellas y, por otro, las reglas de organización y los mecanismos, fenómenos y elementos implicados en ellas.

La didáctica de la conversación es, hoy en día, un campo al que prácticamente no se ha atendido. No obstante, como hemos apuntado con anterioridad, se han publicado algunos trabajos que se centran en la enseñanza de la conversación a partir de cuatro objetivos específicos (cfr. Scarcella 1988: 78 y ss.) * reflexionar sobre la posibilidad de enseñar las características conversacionales explícitamente o de forma indirecta, fomentar y favorecer el uso de materiales lingüísticos auténticos, crear actividades de clase que favorezcan la aparición de contextos de aprendizaje óptimos y programar secuencias de actividades para trabajar la estructuración de la conversación.

En el seno del Análisis de la conversación aplicado a la enseñanza de lenguas segundas o extranjeras, se viene tratando, desde hace ya algunos años, la manera en que debe enseñarse la «competencia conversacional» (Richards 1990). Algunos investigadores afirman y argumentan que es necesario una presentación explícita de las características esenciales de la conversación, seguida de la realización de una gran cantidad de ejercicios variados, para que pueda producirse el desarrollo de la competencia conversacional, pues los alumnos necesitan saber cómo ganar tiempo para pensar (usando marcadores $\mathrm{mmm}$, eeeh, déjame ver...), cómo cambiar de tema (a propósito, fíjate, por otro lado...), cómo parecer interesados en la intervención del hablante (apoyos), etc., antes de trabajar con ello e, incluso, antes de interactuar. Otros investigadores, por el contrario, afirman y argumentan que la competencia conversacional no puede ser enseñada explícitamente, y que, por lo tanto, ha de trabajarse de forma indirecta, basándose, para ello, en el hecho de que la fluidez interactiva que se adquiere en la lengua materna no se enseña ni se aprende en contextos educativos, sino que forma parte del proceso de socialización. Estas dos posturas han dado lugar a la aparición de dos enfoques diferentes en la enseñanza de la conversación (García García 2004 y 2009; Richards 1990):

1. Enfoque indirecto, que considera que lo adecuado es desarrollar la habilidad conversacional a partir de la práctica repetida.

2. Enfoque directo, que cree necesario enseñar explícita o directamente los aspectos estructurales de la conversación en el aula, a través de un proceso concreto determinado por tres fases sucesivas: ilustración (sensibilización a través de muestras reales de cómo se producen los fenómenos conversacionales), interacción (intercambio de opiniones sobre los fenómenos estudiados y su forma de producción y comparación con la lengua materna) e inducción (búsqueda de reglas o mecanismos que regulan la producción de la conversación). 
Como puede apreciarse, la primera de las posturas se basa en la importancia de la práctica y la segunda en la necesidad de entendimiento, observación y comparación. Lo más indicado, en nuestra opinión, es combinar ambos enfoques, pues la práctica es esencial para desarrollar la habilidad conversacional, pero debe recordarse que no es el mismo el proceso de adquisición de la lengua materna que el de una lengua extranjera, por lo que es primordial que se presenten (implícita o explícitamente) los mecanismos, fenómenos y elementos conversacionales en el aula.

Una de las aportaciones más importantes de los analistas de la conversación a la Lingüística Aplicada a la enseñanza de lenguas ha sido concienciar a los profesionales de la necesidad de trabajar siempre con textos reales, tanto en el aula como en la elaboración de materiales y manuales. En la actualidad, contamos ya con un número considerable de corpus lingüísticos que posibilitan y facilitan la utilización de materiales discursivos auténticos, algunos de ellos, incluso, recogidos específicamente para su aplicación a la enseñanza de lenguas extranjeras (Sánchez et alii 1995); no obstante, aún es muy escasa la utilización que se hace de ellos en el ámbito que nos ocupa, debido, fundamentalmente, al esfuerzo y tiempo que requiere.

Las actividades que se realizan en el aula cumplen un cometido fundamental en el aprendizaje de lenguas segundas o extranjeras; por tanto, los profesores deben elaborar una buena programación de las tareas y ser capaces de promover y controlar la participación de los estudiantes en ellas. Los estudios realizados sobre el tema han demostrado que en las actividades pareadas o de grupos reducidos es donde se produce una mayor cantidad de habla y donde se utiliza una más amplia variedad de actos de habla y de usos sociales lingüísticos; es en ellas, además, donde se da la oportunidad de negociar y, por lo tanto, donde se pueden poner en funcionamiento todos los principios y mecanismos estructurales, especialmente cuando dichas actividades implican interacción conversacional. En la programación de tareas ha de tenerse en cuenta, pues, que su realización sea pareada o en grupos reducidos y, obviamente, que sean apropiadas para el nivel de aprendizaje de los estudiantes y lo más breves posible, de forma que no agoten contenidos y no cansen al estudiante; de esta manera se favorece la producción de interacción informal, se multiplica el número de oportunidades que los alumnos tienen de usar la lengua y se mantiene la motivación del aprendiz.

Finalmente, desde hace unos años, se ha comenzado una línea de investigación aplicada a la enseñanza de la conversación en ELE que tiene como cometido ofrecer propuestas de actividades, secuencias de actividades o unidades didácticas para trabajar la estructuración de la conversación en el aula. Estas propuestas, en 
su mayoría presentadas como trabajos de investigación de másteres o como parte de tesis doctorales, toman como base los hallazgos procedentes del Análisis de la conversación y de estudios realizados de forma específica con estudiantes de ELE de distintas lenguas y culturas, por lo que su enfoque es totalmente adecuado.

El primer trabajo en el que se aplicaban los datos procedentes de estudios conversacionales a la didáctica de ELE, de E. García (2003, 2004), presentaba una revisión del tratamiento de los fenómenos conversacionales en manuales y materiales de ELE y una propuesta para la enseñanza de elementos y fenómenos relacionados con el intercambio de turnos de habla que resultaba bastante original y creativa, si bien no seguía ninguna metodología específica.

Los trabajos pioneros de M. García (2004, 2007, 2009) sobre la enseñanza de la conversación sí tienen ya una base metodológica clara, que solo se había probado, hasta entonces, con estudiantes de lenguas extranjeras diferentes del español, y que la autora desarrolla y aplica a ELE. Marta García propone la combinación del enfoque directo y el enfoque indirecto en la elaboración de propuestas didácticas para trabajar turnos de apoyo, reformulación, cambios de tema y sincronización de turnos siguiendo tres fases fundamentales: sensibilización (que aúna las tres etapas propias del enfoque directo: ilustración, interacción, inducción), práctica y revisión y, con ello, sienta las bases metodológicas para el diseño de secuencias de actividades y unidades didácticas para la enseñanza de características conversacionales en ELE.

La propuestas ofrecida por De Mingo Gala (2008-2010), una programación completa de contenidos para un curso de nivel B1 (en el Instituto Cervantes de Estambul), toma como base metodológica la propuesta por Cestero (2005), en lo que respecta tanto al inventario de fenómenos que requieren enseñanza específica como a la metodología de diseño y didáctica de contenidos conversacionales que detallaremos después y que parte de una combinación de métodos directos e indirectos y de los cuatro pasos generales del enfoque comunicativo, y la de Thornbury (2006), basada en tres pasos: toma de conciencia, apropiación y desarrollo de la autonomía. Se trata, sin duda, de un trabajo de cierta envergadura que puede ser muy útil en la enseñanza y que supone un avance en la didáctica de la conversación en ELE.

L. Ambjoern (2008) reflexiona sobre las características conversacionales que requieren enseñanza específica en el aula y aboga por una enseñanza explícita y sistemática de la conversación. Para ello, presenta una propuesta de elaboración de actividades interactivas libres y actividades orientadas a la comprensión y a la producción (con atención a la forma), que tienen de fondo un enfoque centrado 
en el participante y que, sin duda, resultan de gran utilidad en el desarrollo de la competencia conversacional.

Por último, debemos mencionar los trabajos realizados como memoria de investigación para el Máster en Enseñanza de Español como Lengua Extranjera de la Universidad de Alcalá de I. López Sáez de Burgo (2009), R. Sanz (2010) y A. López de Lerma (2011) que se centran, de forma específica, en la didáctica de la conversación en ELE. López Sáez de Burgo (2009) se inclina por el uso del enfoque directo (actividades para la toma de conciencia y el análisis y la reflexión) para tratar en el aula de ELE los aspectos conversacionales de más complejidad y diferenciación transcultural y diseña un modelo de trabajo en la clase basado en tres objetivos que constituyen fases: en primer lugar, análisis de la percepción personal de los estudiantes hacia fenómenos conversacionales (ejemplificado con la interrupción) y comparación con la percepción de los nativos; en segundo lugar, concienciación de los estudiantes extranjeros con respecto a las normas que rigen los procedimientos conversacionales (ejemplificado con el intercambio de turnos), tanto en su lengua y cultura como en la lengua y cultura metas, $y$, por último, evaluación del efecto que el conocimiento produce sobre la percepción de los estudiantes. Toda la propuesta se realiza a partir de grabaciones de conversaciones reales y, sin duda, resulta de gran interés.

Sanz Escudero (2010), por su parte, ofrece una propuesta para enseñar los turnos de apoyo conversacionales a estudiantes de ELE, organizada por niveles, en la que combina, siguiendo las indicaciones de M. García (2004 y 2009) y de Cestero (2005), el enfoque directo e indirecto y establece secuencias de actividades a partir de las siguientes fases: presentación (sensibilización, audición, sistematización), práctica controlada, práctica libre y evaluación. El material fundamental para las actividades es real, conversaciones grabadas para tal fin, lo que constituye, sin duda, un paso adelante en la didáctica de la conversación.

Por último, hemos de mencionar el trabajo de López de Lerma (2011), que parte de una conversación real para trabajar fenómenos y elementos conversacionales que en ella aparecen, a partir de secuencias de actividades, en el aula de ELE (tipos de turnos en cuanto a su forma o función; recursos de toma, mantenimiento y finalización de turno; tácticas de conclusión; forma y función de las interrupciones; forma y función de turnos de apoyo; secuencias de apertura, de cierre y de narración, y organización general de la conversación). La metodología que utiliza es la propuesta por García (2004 y 2009) y por Cestero (205), a partir de cuatro fases (en su caso, presentación, sensibilización, repertorio y práctica), avanzando así en un mismo camino, que, en un futuro próximo, si contamos con 
más trabajos parciales como los mencionados, permitirá ir llenando el vacío existente con respecto a la didáctica de la conversación.

\section{Conclusiones y perspectivas de futuro}

La enseñanza de la conversación, en estos momentos, tiene como objetivo principal que los alumnos sean capaces de expresar y entender ideas en la lengua extranjera que aprenden. Eso es importante y debe seguir potenciándose, pero se debe intentar también que los estudiantes adquieran la competencia conversacional necesaria para interactuar en la lengua meta de forma apropiada y natural, lo que requiere que se atienda a los mecanismos, fenómenos y elementos estructurales de la conversación, tanto en los programas, manuales y materiales de ELE, como en el aula de LE.

Para que ello sea posible, es necesario continuar con los estudios transculturales iniciados en el marco del Análisis de la conversación, y, de manera particular, debe seguir investigándose sobre las características de las conversaciones en ELE de estudiantes con distintas lenguas y culturas. Como hemos mencionado con anterioridad, conviene ampliar el ámbito de investigación con estudiantes procedentes de culturas distintas a las ya tratadas ${ }^{5}$ y temas que aún están por tratar ${ }^{6}$.

Por otro lado, es el momento de ofrecer aportes didácticos. Los manuales y materiales especializados prácticamente no trabajan con las características conversacionales, por lo que resulta imprescindible que los profesionales en ELE hagan propuestas válidas para la enseñanza de los fenómenos, mecanismos y elementos conversacionales. A este respecto, conviene destacar que la enseñanza de las reglas de estructuración de la conversación y los fenómenos y elementos relacionados con ellas debe ser específica y las clases de conversación deben estar programadas para darles cabida, siempre de forma integral, junto con los contenidos gramaticales, léxicos y pragmáticos o nociofuncionales. Los pasos a seguir en la enseñanza de dichas características conversacionales, y, por tanto, la secuenciación adecuada en el diseño de actividades y secuencias de actividades o unidades didácticas, pueden ser los mismos que se reconocen actualmente en las metodologías comunicativas:

1) Presentación de las características conversacionales. El profesor debe presentar las características conversacionales de forma clara y precisa, prestando especial atención a su forma de producción y a su función en la interacción. Tiene que asegurarse de que los alumnos comprenden su uso, su utilidad y su modo de producción antes

5. Hasta el momento, se han tratado las conversaciones de estudiantes alemanes, finlandeses, taiwaneses, británicos e italianos. Están en marcha trabajos sobre conversaciones de estudiantes árabes y japoneses.

6. Como, por ejemplo, marcas de final de turno, secuencias de cierre conversacional, etc. 
de terminar esta fase. En esta primera etapa se trabajarían los tres pasos que propone el método directo mencionado con anterioridad (ilustración, interacción e inducción).

2) Realización de actividades encaminadas al aprendizaje de las características conversacionales. Actividades cerradas y dirigidas totalmente por el profesor, en las que se ejercita de forma individual el uso de las características seleccionadas.

3) Realización de actividades diseñadas para reforzar el aprendizaje de las características conversacionales. Actividades semicerradas y dirigidas totalmente por el profesor, en las que se ejercita el uso interactivo, diádico o en grupos reducidos, de las características trabajadas.

4) Realización de actividades para conseguir la adquisición de las características conversacionales. Actividades abiertas y semidirigidas por el profesor, en las que los alumnos deberán poner en práctica, en interacciones más o menos naturales y de forma espontánea, las características aprendidas.

Los contenidos conversacionales se tienen que introducir de forma gradual, atendiendo, por un lado, a la simplicidad y complejidad de producción y, por otro, al mayor o menor rendimiento funcional. En cada curso completo se deberá trabajar con aspectos de todos los niveles estructurales, comenzando por los pertenecientes a la estructura pormenorizada y progresando hasta llegar a los de la estructura general, pues de nada sirve hacer que los estudiantes produzcan una conversación completa, si no saben finalizar un turno, apoyar, narrar o construir una secuencia. Ha de presentarse siempre la función que cumplen los mecanismos, fenómenos o elementos en la producción y estructuración de la conversación, su forma más frecuente de realización y tipos de estructura sintáctico-semántica y vocabulario específico, en caso de necesitarse, sin olvidar que la mejor manera de trabajar los elementos estructurales relativos a la producción es en forma de estrategias: estrategias para concluir un turno, estrategias para tomar la palabra, estrategias para mostrar seguimiento, estrategias para cambiar de tema, etc.

Ahora bien, para realizar propuestas didácticas y crear materiales que permitan trabajar la conversación en el aula de ELE, es necesario contar con materiales reales, conversaciones naturales, a partir de las cuales se elaboren actividades y secuencias de actividades, así como unidades didácticas. En estos momentos, no existe ningún corpus específico de libre disposición. Pueden utilizarse los materiales que ofrecen los corpus de la Real Academia Española y del grupo Val.Es.Co. o de la Universidad de Alcalá, pero no son materiales específicos. Es el momento, pues, de emprender la recogida de un corpus de conversaciones ( $y$ otros tipos de actividades interactivas) para la enseñanza de ELE, que 
cuente con interacciones conversacionales producidas en diferentes contextos y en las que participen personas con distintas características sociales (sexo, edad, nivel de instrucción, etc.).

Confiamos en que las ideas y lo datos ofrecidos aquí sobre el funcionamiento de la conversación, sobre su estudio en el marco de la enseñanza de español como lengua extranjera y sobre la elaboración de propuestas didácticas para su enseñanza animen a muchos profesionales a unirse a esta línea de investigación, de manera que, en un futuro mediato, se posibilite y favorezca el desarrollo de la enseñanza de la conversación en ELE.

\section{Referencias bibliográficas}

Albert, E. M. (1972). «Culture patterning of speech behavior in Burundi». En: J. Gumperz y D. Hymes. (eds.). Directions in Sociolinguistics. New York. Holt, Rinehart \& Winston: 72-105.

Álvarez Martínez, M. A.; De la Fuente Martínez, M. V; Giraldo Silverio, I.; Martín Martín, F., Sanz Sánchez, B. y Torrens Álvarez, M. J. (2001). Sueña 3. Madrid. Universidad de Alcalá-Anaya.

Álvarez Tejedor, A.; Aguilar López, A. M. y Simarro Vázquez, M. (2010). Hablamos español (nivel C). León. Everest.

Ambjoen, L. (2008). «Enseñanza y aprendizaje de la competencia conversacional en español: planteamientos del problema y propuestas de solución». En: RedELE. 13. <http://www.educacion.es/redele/Revista13/ensenanzaapren-ambjoern.pdf $>$.

Bilmes, J. (1992). «Dividing the rice: A microanalysis of the mediator's role in a Northern Thai negotiation». Language in society. 21: 569-601.

Blanco Canales, A.; Fernández lópez, M. C. y Torrens Álvarez, M. J. (2001). Sueña 4. Madrid. Universidad de Alcalá-Anaya.

Briz Gómez, A. (1996). El español coloquial: situación y uso. Madrid. Arco/Libros.

- (1998). El español coloquial en la conversación. Esbozo de pragmagramática. Barcelona. Ariel.

Castro, F; Martín, F.; Morales, R. y Rosa, S. (1990). Ven 1. Madrid. Edelsa/Edi6.

Castro, F., y Rosa, S. (1992). Ven 3. Madrid. Edelsa/Edi6.

Cerrolaza, M.; Cerrolaza, O.y Llovet, B. (1998).Planet@.ElE 1. Madrid. Edelsa. 
- (1999).Planet@.ELE 2. Madrid.Edelsa.

Cestero Mancera, A. M. (2000a). El intercambio de turnos de habla en la conversación. Análisis sociolingüístico. Alcalá de Henares. Servicio de Publicaciones de la Universidad de Alcalá.

Cestero Mancera, A. M. (2000b). Los turnos de apoyo conversacionales. Cádiz. Servicio de Publicaciones de la Universidad de Cádiz.

- (2005). Conversación y enseñanza de lenguas extranjeras. Madrid. Arco/Libros.

Consejo de Europa (2002). Marco común europeo de referencia para las lenguas: aprendizaje, enseñanza, evaluación. Madrid. Ministerio de Educación, Cultura y Deporte. Secretaría General Técnica del MECD-Subdireción General de Informaciones y Publicaciones y Grupo ANAYA.

Daden, I. (1982). Bargaining in a Guatemalan Highland Quiche-Mayan Market. Tesis doctoral inédita. California. University of California at Los Angeles.

De Mingo Gala, J. A. (2008). La enseñanza de la conversación en el aula de ELE. Propuesta de contenidos para el curso de conversación intermedio (B1) del Instituto Cervantes de Estambul. Memoria de máster del Instituto Cervantes. Madrid. MarcoELE. 10 (2010).

ElLis, R+ (1999). «Theoretical perspectives on interaction and language learning». En: R. Ellis et alii Learning a Second Language through Interaction. Amsterdam. Benjamin: 3-31.

Fente, R.; Fernández, J. y Siles, J. (1982). Curso intensivo de español. Ejercicios prácticos. Iniciación y Elemental. Madrid. SGEL.

- (1990). Curso intensivo de español. Ejercicios prácticos. Intermedio y superior. Madrid. SGEL.

Fernández Cinto, J. (1991). Actos de habla de la lengua española. Repertorio. Madrid. Edelsa.

Fernández, M. J. y Albelda, M. (2008). La enseñanza de la conversación coloquial. Madrid. Arco/Libros.

Gallardo Paúls, B. (1993). Lingüistica perceptiva y conversación: secuencias. Minneapolis-Valencia. University of Minnesota-Universitat de València. (Lynx, annexa 4).

- (1996). Análisis conversacional y pragmática del receptor. Valencia. Episteme.

- (1998). Comentario de textos conversacionales I. De la teoría al comentario. Madrid. Arco/Libros. 
García Castro, E. (2003). El intercambio de turnos de babla en la conversación española y su enseñanza en la clase de español como lengua extranjera. Memoria de máster inédita. Alcalá de Henares. Universidad de Alcalá.

- (2004). «El intercambio de turnos de habla y su enseñanza en la clase de español como lengua extranjera». Cuadernos Cervantes. 53: 40-44.

García García, M. (2004)+ «La conversación en clase‡ una propuesta de enseñanza del discurso conversacional para el aula de E/LE». Glosas Didácticas. Disponible en: http://www.um.es/glosasdidacticas/doc-es/GD12/07 garcia.pdf

- (2005). «La competencia conversacional en los estudiantes de español como lengua extranjera». Lingüística en la Red. II. Disponible en: <http://www. linred.com>.

- (2007). «La enseñanza de la competencia conversacional». Frecuencia L. Revista de Didáctica del Español Lengua Extranjera. 33: 16-21.

- (2009). La competencia conversacional de estudiantes de español como lengua extranjera: análisis y propuesta didáctica. Tesis doctoral inédita. Alcalá de Henares. Universidad de Alcalá.

Gardner, R. (1994)+ "Conversation analysis: Some thoughts on its applicability to applied linguistics». Australian Review of Applied Linguistics. 11: 97-118.

- (1997). «The listener and minimal responses in conversational interaction». Prospect. 12 (2): 12-32.

Gardner, R. y Wagner, J. (eds.) (2004). Second Language Conversations. London. Continuum.

Gelabert, M. J*; Herrera, M.; Martinell, E. y Martinell, F. (1990). Repertorio de funciones comunicativas del español. Niveles umbral, intermedio $y$ avanzado. Madrid. SGEL.

НАтсн, Е. (1978). «Discourse analysis and second language acquisition». Enः E. Натсн (ed.). Second Language Acquisition, A Book of Readings. Rowley. MA. Newbury House: 306-326.

Haviland, J. (1977). Reputation, and Knowledge in Zinacantan. Chicago. University of Chicago Press.

Hayashi, R. (1991). «Floor structure of English and Japanese conversation». Journal of Pragmatics, 16:1-30.

Hopper, R. (1990-91). «Ethnography and Conversation Analysis after Talking Culture». Research on Language and Social Interaction. 24: 161-170. 
INGLÉs, B. (2010). El funcionamiento de los turnos de apoyo en la conversación en español de estudiantes ingleses de ELE. Trabajo de investigación tutelado inédito. Madrid. Universidad Nebrija.

Instituto Cervantes (2006). Plan curricular del Instituto Cervantes. Niveles de referencia para el español. Madrid. Instituto Cervantes/Biblioteca Nueva.

Jefferson, G. (ed.) (1992). H. Sacks, Lectures on Conversation. Oxford. Basil Blackwell.

Levinson, S. C. (1989). Pragmática. Barcelona. Teide.

López de Lerma Díez, A. (2011). La enseñanza de la conversación en el aula E/ LE. Memoria de máster inédita. Alcalá de Henares. Universidad de Alcalá.

López Sáez de Burgo, I. (2009). La enseñanza del intercambio de turnos en la conversación española. Memoria de máster inédita. Alcalá de Henares. Universidad de Alcalá.

Martínez, M. C. (2009). La organización temática en la conversación de estudiantes finlandeses de español como lengua extranjera. Trabajo de investigación tutelado inédito. Madrid. Universidad Nebrija.

MaYnARD, S. K. (1990). «Conversation management in contrast: Listener response in Japanese and American English». Journal of Pragmatics. 14: 397 412.

Moerman, M. (1972). «Analysis of Tai conversation: Making accounts, finding breacher and taking sides». En: D. Sudnow (ed.). Studies in Social Interaction. New York. The Free Press: 170-228.

- (1977). «The preference for self-correction in a Tai conversational corpus». Language. 53: 872-882.

- (1988). Talking Culture. Ethnography and Conversation Analysis. Philadelphia. University of Pennsylvania Press.

Pascual, C. (2011). Los turnos de apoyo en la conversación de estudiantes italianos de E/LE. Memoria de máster inédita. Madrid. Universidad Nebrija.

Pérez Ruiz, J. (2009). Los turnos de apoyo en la conversación de estudiantes taiwaneses de ELE. Trabajo de investigación tutelado inédito. Madrid. Universidad Antonio de Nebrija.

- (2011). El funcionamiento de los turnos de apoyo en la conversación de estudiantes taiwaneses de ELE. Tesis doctoral inédita. Madrid. Universidad Nebrija.

Philips, L. (1976). «Some source of cultural variability in the regulation of talk». Language in Society. 5: 81-87. 
Richards, J. C. (1990). The Language Teaching Matrix. Cambridge. Cambridge University Press.

Ridruejo, E.; Hoyos, C; Mendizábal de la Cruz, N. y Vela Delfa, C. (2010). Hablamos español (nivel B2). León. Everest.

Riggenbach, H. (1991). «Toward an understanding of fluencyः A microanalysis of non-native speaker conversations». Discourse Processes. 14: 423-441.

- (ed.) (2000). Perspectives on Fluency. Michigan. University of Michigan Press.

Rubio, M. (2007). «Contenidos para la clase de conversación en ELE. Propuesta de un programa de estudios a partir de la estructura conversacional». Frecuencia L. Revista de Didáctica del Español Lengua Extranjera. 33: 9-15.

- (2008). La interrupción y la superposición de babla en la conversación de estudiantes taiwaneses de español como lengua extranjera. Trabajo de investigación tutelado inédito. Madrid. Universidad Nebrija.

Sacks, H.; Schegloff, E. y Jefferson, G. (1974). «The simplest systematics for the organization of turn taking for conversation». Language. 50: 696-731.

Sánchez, A. et alii (1995). Cumbre. Corpus lingüístico del español contemporáneo. Fundamentos, metodología y aplicaciones. Madrid. SGEL.

SANz, R. (2010). La enseñanza de los turnos de apoyo en la conversación española. Memoria de máster inédita. Alcalá de Henares. Universidad de Alcalá.

Scarcella, R. (1983). «Developmental trends in the acquisition of conversational competence by adult second language learners». En: J. Wolfson, ed. Sociolinguistics and Language Acquisition. Rowley. Newbury House: 175-183.

- (1988). «Conversation analysis in L2 acquisition and teaching». Annual Review of Applied Linguistics. 9: 72-91.

Schiffrin, D. (1994). Approaches to Discourse. Oxford. Basil Blackwell.

Stewner-Manzanares, G. J. (1983). Turn-taking Structure of Second Language Learners of Spanish at Different Oral Proficiency Levels. Tesis doctoral inédita. Washington. Georgetown University.

TAnnen, D. (1983). «When is an overlap not an interruption? One component of Conversational Style». En* R. Di Pietro, W. Frawley y A. Wedel (eds*). The First Delaware Symposium on Language Studies. Newark, D. E. University of Delaware Press: 119-129.

- (1987). «Conversational style ». En: H. W. Dechert y M. Raupach (eds.). Psycholinguistic Models of Production. N.J. Norwood. Ablex: 251-267. 
TaO, H. y Thompson, S. A. (1991). «English backchannels in Mandarin conversations: A case study of superstratum pragmatic 'interference'». Journal of Pragmatics. 16: 209-223.

Thornbury, S. (2006). How to Teach Speaking. Harlow. Pearson Education Limited.

Tusón Valls, A. (1997). Análisis de la conversación. Barcelona. Ariel.

Varela, S. (dir.) (1994). Tácticas de conversación. ELE. Madrid. SM.

VÁzquez, G. (2000). La destreza oral. Madrid. EDELSA. 